\title{
DO YOU KNOW?
}

The National Aboriginal Education Committee (NAEC) advises the Minister for Education and Youth Affairs, her department and other appropriate authorities, on the development of all levels of education for Aboriginal people. It is the vehicle of government policy on Aboriginal education.

NAEC members are Aborigines or Torres Strait Islanders and their appointments follow Australia-wide consultation with significant Aboriginal groups, including the NAEC, the National Aboriginal Conference, and the State Aboriginal Education Consultative Groups. Members of the current NAEC are:

Chairman:

Deputy Chairman:

Executive Officer:

Research Officer:

Information Officer:

Secretary to Chairman:
Paul Hughes

Errol West

Kaye Price

Vic Forrest

George Ahmat

Wendy Fenton
P.0.Box 826

Woden ACT 2606

Telephone 062.897204

NEW SOUTH WALES

Mr Charles Dixon, 7/188 Campbell Street, Darlinghurst, NSW 2010. Telephone, 02.292268 (work) 02.334588 (home)

Mr Robert Morgan, Chairman, NSW Aboriginal Education Consultative Group, Aboriginal Education Unit, Department of Education, Box 33 GPO, Sydney, NSW, 2001. Te1: 02.2408870 (work) 02.7993477 (home).

Mrs Davina Tyrre11, 68 Albert Street, Erskineville, NSW, 2043. Te1. 02.2408819 (work) 02.5192390 (home). (Specialist: Curriculum Development).

\section{VICTORIA}

Mrs Mary Atkinson, 186 Hayes Street, Shepparton, Vic 3630. Tel. 058.2161111.

Ms Eve Fesl, Director, Aboriginal Research Unit, Monash University, 4th Floor Education Building, Wellington Road, Clayton, Vic 3168. Tel. 03.5410811 (work) 03.8771228 (home). (Specialist: Language Deve (opment). 


\section{QUEENSLAND}

Ms Helena Gulash, 50 Griffiths Street, South Townsville, Q1d 4810. Tel. 077.722944 (work). (Specialist: Primary/Secondary Education.)

Mr Eric Law, 1 Marbella Street, Slacks Creek, Q1d 4127. Te1. 07.2248171 (work) 07.2094361 (home).

Mrs Ethel Munn, Pegler Street, Quilpie, Q1d 4480. Tel. Quilpie 169.

Mrs Sepi Woosup, C/- Post Office, Bamaga, Q1d 4876.

Te1.070.743147 (work) 070.5132888 (Cairns Office).

\section{SOUTH AUSTRALIA}

Ms Wendy Clinch, 3 Lethbridge Court, Port Augusta, SA 5700. Te1. 086.424099 (work) 086.423429 (alternative contact no.)

Mr Peter Zweck1, 37a Roberts Street, Croydon, SA 5008. $\mathrm{Te} 1.08 .2282245$ (work) 08.459698 (home).

NORTHERN TERRITORY

Mr Rex Granites (Japanangka), Yuendumu, Via Alice Springs NT 5751. Tel. Radio Telephone 447. (Specialist: Traditional Education).

Ms Wendy Ludwig, Aboriginal Task Force, Darwin Community College, P.O.Box 40146, Darwin, NT 5792. Te1. 089.201339

Mrs Didamain Uibo, C/- Post Officer, Batchelor, NT 5791. Te1. 089.760041.

WESTERN AUSTRALIA

Mr Stephen Albert, P.0. Box 50, Broome, WA 6725. Te1. 091.921010 (messages between 12 noon - 3 p.m. EST) 091.921662 (Broome Office).

Mrs Oriel Green, 33 Allinson Drive, Girrawheen, WA 6064. Te1. 09.4204548 (work). (Specialist: Early Childhood Education).

\section{TASMANIA}

Mr Laurie Padmore, 12 Gatty P1ace, Devonport, Tas 7310. Tel. 004.246522 (home) 004.242495 (work - Mon., Wed., Fri., 9 a.m. 10 a.m.; 3 p.m. - 5 p.m.) 004.302280 (work - Tues. \& Thurs. 